\title{
Cookies Internal Temperature Estimation Using IHCP Methodology
}

\author{
Eduardo Macías Ávila* and Yaneth Aguilar Díaz
}

\author{
CIDESI, Alianza Sur No. 203, Autopista aeropuerto km 10, Apodaca, N.L. México
}

\begin{abstract}
This work constitutes a continuation of previous work on determining the internal temperature of foods by applying inverse heat transfer problem solving techniques. In this research, a mathematical model of heat transfer applied to foods is developed whose central part heat transfer can be described in Cartesian coordinates. This model uses techniques established in the previous work. The technique is based on the adjustment of parameters involved in heat transfer, minimizing the sum of squared errors between the measured temperatures and those calculated by the mathematical model. This paper discusses how the precision of this method would be affected with respect to the measurement time of the surface temperature and the delay time of the measurement.
\end{abstract}

Keywords: Inverse problem, Heat transfer, Inner temperature estimation, Food processing, Infrared thermography.

\section{INTRODUCTION}

In this research, a method is developed to determine the core temperature based on the surface temperature vs time curve, obtained by means of infrared thermography. The normal procedure for internal temperature measurement is to insert a thermocouple into the part, but that would lead to partial destruction of the object and cannot be done for a large set of objects.

In the food industry, temperature control of food products is essential. Both lack of cooking and overcooking have negative consequences. Foods that lack cooking can cause illness in consumers, whether the increase in temperature or residence time is not enough, in order to kill harmful bacteria or parasites, especially at the inner part of the product [1-3]. On the other hand, an overheating affects the taste and texture of the food, due to excessive loss of moisture, and the possible destruction of certain nutrients, which leads to a nutritional reduction of the food [1-3].

In addition to the above, there are also industrial processes where the use of invasive techniques such as the insertion of thermocouples is not possible, as is the case of polymer processing [4, 5], in which the product is formed by the polymer solidification.

A measurement of the surface temperature by means of thermography does not have these problems, although in this case the internal temperature is not being measured. In order to obtain the internal temperature, it is necessary to obtain the correlation between these two temperatures.

"Address correspondence to this author at the CIDESI, Alianza Sur No. 203, Autopista aeropuerto km 10, Apodaca, N.L. México;

Tel: 52(81)1493 5550;

E-mail: eduardo.macias@cidesi.edu.mx; yaneth.aguilar@cidesi.edu.mx
The following equation shows based on which parameters the internal temperature can be determined; from the surface temperature curve, time, as well as the geometry of the piece, thermal properties of the materials and cooling conditions.

$T_{c}=F\left[\overrightarrow{T_{s}}, \bar{t}\right.$, Geometry, cooling conditions, thermal properties $]$

Different approaches have been developed to obtain this correlation: Juan G. Ibarra et al. [2] estimated the internal temperature in chicken meat by means of external temperature measurements using a multilayer neural network. However, they recognized that geometric data are important in determining inner temperature and that variable shapes and thicknesses may directly affect the heat transfer process in meats. In a later work [3] Yan Tao et al. incorporate a laser range system with an IR imaging system to extract the 3D information of the chicken breast, involving the effect of these parameters within a neural network. Neural network training requires a large number of experiments to adjust for valuable factors in the network, which becomes problematic when a parameter not considered is changed such as a different cooling coefficient or cooking of food with different thermal properties. An option to have a lower number of experimental trials to adjust the parameters is a model based on fundamental laws.

It is possible to obtain this correlation from the solution of the heat transfer equation. This would allow include the effect of parameters on heat transfer and reducing the number of experiments to adjust the variables. It should be clarified that the analytical solution can be difficult to obtain due to the complex geometry of the part, heat transfer in various directions, temperature-dependent properties, non-linear heat 
transfer conditions at the boundaries and uncertain initial condition. Even so, there are certain processes where it is possible to pose the problem of determining the internal temperature correlation through an analytical model, taking advantage of certain details of the process, for example considering that the heat flow is carried out preferably in one direction due to part dimensions, constant thermophysical properties, or linear cooling conditions.

Works have been reported, although not related to the food industry, where the internal temperature is obtained from the surface temperature curve measured by thermography. Bendada and K. T. Nguyen [4] developed a mathematical model to determine the initial temperature distribution using the surface temperature in a polymer processing. They considered unidimensional heat transfer in a piece with flat geometry. A patent [5] describes a method for estimating the temperature distribution throughout the thickness of a preform in blow molding process by measuring the surface temperature. Dean Anthony et al. [6] present a technique to determine the internal temperature based on the measurement of surface temperature using infrared thermography on a thermal test cell.

Determining the internal temperature profile belongs to a class of problems known as IHCP. In this class of problems, parameters such as internal condition, heat flow, thermophysical properties, etc. are determined from a measured temperature profile. With the use of IHCP techniques, it is sought to obtain these parameters complying with the fundamental laws of heat transfer. Ozisik and Orlande [7] give a summary of different techniques used to solve inverse heat transfer problems. Such techniques generally require the solution of the associated direct problem. Difficulties encountered in the solution of IHCP should be recognized. IHCP are mathematically classified as illposed in a general sense, because their solutions may become unstable, measurement errors can be amplified if proper care is not taken, as a result of the errors inherent to the measurements used in the analysis.

This work is a continuation of a previous investigation [8] in which a method was developed to determine the internal temperature of foods with cylindrical geometry based on surface temperature measurements by means of infrared thermography. The determination of internal food temperature was obtained by applying inverse heat conduction problem solving techniques. In this research, the developed method is extended to include foods whose heat transfer can, as in the previous case, be modeled with simple partial differential equations. In this case, the heat transfer equation is solved in Cartesian coordinates, where in the central part the heat transfer is preferably carried out in one direction, which is along the thickness of the food. In this investigation also considered the effect of a plate that supports the food and the interface thermal resistance between the food and the plate.

\section{MATHEMATICAL DEVELOPMENT}

\subsection{Thermal Model Approach}

The first thing to do is solve the heat conservation equation described in Cartesian coordinates. This is an equation that describes conduction heat transfer in solid bodies.

There are several assumptions that are being made in the model:

1) The heat is transferred through the $x$ axis (thickness).

2) The effects of the borders on the product are neglected, it is expected that this assumption is more valid in the core of the product.

3) The thermophysical properties are constant.

We considered the following geometry with the following materials, food and baking silicon. The scheme in Figure 1 shows the arrangement of both materials. Heat is transferred through both the upper and lower surfaces. The two materials have different thermophysical properties. It is also considered that there is an interfacial thermal resistance at the contact surface between both materials.

\begin{tabular}{|c|}
\hline Cookie \\
\hline Baking silicon \\
\hline
\end{tabular}

Figure 1: Scheme of materials in the domain of study.

Therefore, the equation to be solved is as follows:

$\frac{\partial}{\partial x}\left(k_{i} \frac{\partial T}{\partial x}\right)=\rho_{i} C_{P i} \frac{\partial T}{\partial t}$ 
Where $k$ is the thermal conductivity, $\rho$ is the density and $C_{P}$ is the specific heat. The subscript " $i$ " indicates the type of material depending on the calculation area.

\subsection{Boundary Conditions}

In this partial differential equation, it is necessary to establish the initial conditions and boundary conditions.

With the boundary conditions given by:

Contact surface of the environment with the silicon plate

$x=0 \quad-k_{1} \frac{\partial T}{\partial x}=h_{1}\left(T_{\infty}-T\right)$

Contact surface of the environment with the food:

$x=L \quad-k_{2} \frac{\partial T}{\partial x}=h_{2}\left(T-T_{\infty}\right)$

At the interface between the two materials, continuity in the heat flow is assumed, so it is necessary to:

$$
\begin{aligned}
x & =l \\
-\left.k_{1} \frac{\partial T}{\partial x}\right|_{\text {silicon }} & =-\left.k_{2} \frac{\partial T}{\partial x}\right|_{\text {food }}
\end{aligned}
$$

In this model, it is not considered that there is continuity in the temperature profile at the interface, but rather there is an interfacial thermal resistance between the product and the silicone plate.

$$
\begin{gathered}
x=l \\
-\left.k_{1} \frac{\partial T}{\partial x}\right|_{\text {silicon }}=-\left.k_{2} \frac{\partial T}{\partial x}\right|_{\text {food }}=\frac{\left.T\right|_{\text {silicon }}-\left.T\right|_{\text {food }}}{R_{\text {th }}}
\end{gathered}
$$

The solution of the equation is expressed as a sum of functions:

$T(x, t)=T_{\infty}+\sum_{n=1}^{\infty} A_{n} \theta_{t}^{n}(t) \theta_{n, i}(x)$

If this solution is substituted in the equation for the conservation of heat, we obtain:

$$
\frac{1}{\theta_{t}^{n}(t)} \frac{\partial \theta_{t}^{n}(t)}{\partial t}=\frac{\alpha_{i}}{\theta_{n, i}(x)} \frac{\partial^{2} \theta_{n, i}(x)}{\partial x^{2}}
$$

Here each of the functions remains separately on each side of the equation. For this to happen, the only way (since the term on the left side is a function of time and the right side is a function of $\mathrm{x}$-axis), is that they are equal to a constant. The left side of the equation 8 looks like this (the constant has to be negative, since a positive value would give an increasing exponential expression over time, something that is physically not correct).

$\frac{1}{\theta_{t}^{n}(t)} \frac{\partial \theta_{t}^{n}(t)}{\partial t}=-\lambda_{n}^{2}$

Solving it we arrive at an exponential function with respect to time:

$\theta_{t}^{n}(t)=e^{-\lambda_{n}^{2} t}$

This expression is valid for the two domains of interest: on the side of the silicone plate and on the one corresponding to the food.

The right side of the equation 8 remains:

$\frac{\partial^{2} \theta_{n, i}(x)}{\partial x^{2}}=-\frac{\lambda_{n}^{2}}{\alpha_{i}} \theta_{n, i}(x)$

The solution for the function is obtained as:

$\theta_{n, i}(x)=a_{n, i} \cos \left(\frac{\lambda_{n}}{\sqrt{\alpha_{i}}} x\right)+b_{n, i} \sin \left(\frac{\lambda_{n}}{\sqrt{\alpha_{i}}} x\right)$

The equation (12) can be expressed in a more compact form as follows:

$\theta_{n, i}(x)=r_{n, i} \sin \left(\frac{\lambda_{n}}{\sqrt{\alpha_{i}}} x+\phi_{n, i}\right)$

The solution of the heat conservation equation is in terms of the sines and cosines functions, as we have in the formulas.

$0 \leq x<l$
$T(x, t)=T_{\infty}+\sum_{n=1}^{\infty} A_{n} e^{-\lambda_{n}^{2} t} r_{n, 1} \sin \left(\frac{\lambda_{n}}{\sqrt{\alpha_{1}}} x+\phi_{n, 1}\right)$

$l<x \leq L$
$T(x, t)=T_{\infty}+\sum_{n=1}^{\infty} A_{n} e^{-\lambda_{n}^{2} t} r_{n, 2} \sin \left(\frac{\lambda_{n}}{\sqrt{\alpha_{2}}} x+\phi_{n, 2}\right)$

This equation is obtained without even considering the boundary conditions and the initial condition.

Let's see next what happens when we include the boundary conditions.

Boundary conditions: 
1) In the inferior surface of the silicon region you have a convection heat flow:

$-k_{1} \frac{\partial \theta_{n, 1}(x)}{\partial x}=-h_{1} \theta_{n, 1}(x) \quad$ for $\quad x=0$

2) On the outside of the product there is a convection heat flow

$-k_{2} \frac{\partial \theta_{n, 2}(x)}{\partial x}=h_{2} \theta_{n, 2}(x) \quad$ for $\quad x=L$

And in the interface

$$
\begin{gathered}
x=l \\
-k_{1} \frac{\partial \theta_{n, 1}(x)}{\partial x}=-k_{2} \frac{\partial \theta_{n, 2}(x)}{\partial x}=\frac{\left(\theta_{n, 1}(x)-\theta_{n, 2}(x)\right)}{R_{t h}}
\end{gathered}
$$

Using the following Biot numbers

$$
\begin{aligned}
& B i_{1}=\frac{h_{1}}{k_{1}} L \\
& B i_{2}=\frac{h_{2}}{k_{2}} L
\end{aligned}
$$

In the next part of the approach we will use the following equation:

$\phi=\frac{\lambda_{n}}{\sqrt{\alpha_{1}} L}$

From equation number 15 it can be obtained:

$\tan \phi_{n, 1}=\frac{\phi}{B i_{1}}$

From equation number 16 , it can be obtained:

$\tan \left(\phi \sqrt{\frac{\alpha_{1}}{\alpha_{2}}}+\phi_{n, 2}\right)=-\sqrt{\frac{\alpha_{1}}{\alpha_{2}}} \frac{\phi}{B i_{2}}$

From this equation it is possible to obtain the tangent value of if the trigonometric identity of the tangent of the sum of two angles is used.

$\tan \phi_{n, 2}=\frac{\tan \left(\phi \sqrt{\frac{\alpha_{1}}{\alpha_{2}}}\right)+\sqrt{\frac{\alpha_{1}}{\alpha_{2} B i_{2}}}}{\sqrt{\frac{\alpha_{1}}{\alpha_{2} B i_{2}}} \tan \left(\phi \sqrt{\frac{\alpha_{1}}{\alpha_{2}}}\right)-1}$

We are going to use the following expressions

$$
\begin{aligned}
& r_{1}=1 \\
& r_{2}=R
\end{aligned}
$$

Using the equation in expression number 17 it is possible to arrive at the following expressions. First, for the heat transfer continuity condition at the interface, the following equation can be reached.

$$
R=\sqrt{\frac{k_{1} \rho_{1} C_{P 1}}{k_{2} \rho_{2} C_{P 2}}} \frac{\cos \left(\phi_{\frac{L}{L}}^{l}+\phi_{n, 1}\right)}{\cos \left(\phi \frac{l}{L} \sqrt{\frac{\alpha_{1}}{\alpha_{2}}}+\phi_{n, 2}\right)}
$$

For the case of discontinuity in the temperature profile due to interfacial resistance, we have:

$$
\sin \left(\frac{\lambda_{n}}{\sqrt{\alpha_{1}}} l+\phi_{n, 1}\right)=R\left[\sin \left(\frac{\lambda_{n}}{\sqrt{\alpha_{2}}} l+\phi_{n, 2}\right)-\frac{k_{2} R_{t h}}{\sqrt{\alpha_{2}}} \cos \left(\frac{\lambda_{n}}{\sqrt{\alpha_{2}}} l+\phi_{n, 2}\right)\right]
$$

From here it is possible to arrive at the following equation:

$\tan \left(\phi \frac{l}{L}+\phi_{n, 1}\right)=\sqrt{\frac{k_{1} \rho_{1} C_{P 1}}{k_{2} \rho_{2} C_{P 2}}} \tan \left(\phi \frac{l}{L} \sqrt{\frac{\alpha_{1}}{\alpha_{2}}}+\phi_{n, 2}\right)-R_{t h} \sqrt{k_{1} \rho_{1} C_{P 1}}$

Developing this expression

$$
\begin{aligned}
& \frac{\tan \left(\phi \frac{l}{L}\right)+\frac{\phi}{B i_{1}}}{1-\frac{\phi}{B i_{1}} \tan \left(\phi \frac{l}{L}\right)}=
\end{aligned}
$$

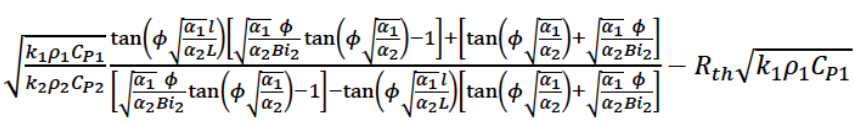

From which it is possible to obtain by means of numerical analysis the value of $\phi$.

\subsection{Initial Conditions}

The functions given in equation 7 are shown to form an orthogonal system of functions. The expression can be set as follows:

$$
-\lambda_{n}^{2} \int_{0}^{L} \rho C_{P} \theta_{n}(x) \theta_{m}(x) d x=\int_{0}^{L} \frac{\partial}{\partial x}\left(k \frac{\partial \theta_{n}(x)}{\partial x}\right) \theta_{m}(x) d x
$$

The integral can be divided as:

$$
\begin{aligned}
& \int_{0}^{L} \frac{\partial}{\partial x}\left(k \frac{\partial \theta_{n}(x)}{\partial x}\right) \theta_{m}(x) d x= \\
& \int_{0}^{l} \frac{\partial}{\partial x}\left(k \frac{\partial \theta_{n}(x)}{\partial x}\right) \theta_{m}(x) d x+\int_{l}^{L} \frac{\partial}{\partial x}\left(k \frac{\partial \theta_{n}(x)}{\partial x}\right) \theta_{m}(x) d x
\end{aligned}
$$

Using integration by parts in the first integral on the right side, we would arrive at: 
$\int_{0}^{l} \frac{\partial}{\partial x}\left(k \frac{\partial \theta_{n}(x)}{\partial x}\right) \theta_{m}(x) d x=$

$\left.k \frac{\partial \theta_{n}(x)}{\partial x} \theta_{m}(x)\right|_{x=0} ^{x=l}-\left.k \frac{\partial \theta_{m}(x)}{\partial x} \theta_{n}(x)\right|_{x=0} ^{x=l}+\int_{0}^{l} \theta_{n}(x) \frac{\partial}{\partial x}\left(k \frac{\partial \theta_{m}(x)}{\partial x}\right) d x$

Which can be rewritten as:

$\left(\lambda_{m}^{2}-\lambda_{n}^{2}\right) \int_{0}^{l} \rho C_{P} \theta_{n}(x) \theta_{m}(x) d x=$

$\left.k \frac{\partial \theta_{n}(x)}{\partial x} \theta_{m}(x)\right|_{x=0} ^{x=l}-\left.k \frac{\partial \theta_{m}(x)}{\partial x} \theta_{n}(x)\right|_{x=0} ^{x=l}$

For the other integral we would have something similar, then for the total integral it would be as:

$\left(\lambda_{m}^{2}-\lambda_{n}^{2}\right) \int_{0}^{L} \rho C_{P} \theta_{n}(x) \theta_{m}(x) d x=$

$\left.k \frac{\partial \theta_{n}(x)}{\partial x} \theta_{m}(x)\right|_{x=0} ^{x=l}-\left.k \frac{\partial \theta_{m}(x)}{\partial x} \theta_{n}(x)\right|_{x=0} ^{x=l}+\left.k \frac{\partial \theta_{n}(x)}{\partial x} \theta_{m}(x)\right|_{x=l} ^{x=L}-\left.k \frac{\partial \theta_{m}(x)}{\partial x} \theta_{n}(x)\right|_{x=l} ^{x=L}$

This equation can be expressed as (after using the boundary conditions 15 and 16)

$\left(\lambda_{m}^{2}-\lambda_{n}^{2}\right) \int_{0}^{L} \rho C_{P} \theta_{n}(x) \theta_{m}(x) d x=$

$k_{1} \frac{\partial \theta_{n, 1}(l)}{\partial x}\left[\theta_{m, 1}(l)-\theta_{m, 2}(l)\right]+k_{1} \frac{\partial \theta_{m, 1}(l)}{\partial x}\left[\theta_{n, 2}(l)-\theta_{n, 1}(l)\right]$

Using equation 17 it can be obtained, knowing that $\lambda_{m}^{2} \neq \lambda_{n}^{2}$

$\int_{0}^{L} \rho C_{P} \theta_{n}(x) \theta_{m}(x) d x=0$

For initial time we would have:

$T_{0}(x)=T_{\infty}+\sum_{i=0}^{+\infty} A_{n} \theta_{n}(x)$

It is possible to obtain the values of $A_{n}$ using the above equation.

$A_{n}=\frac{\int_{0}^{L} \rho C_{P} \theta_{n}(x)\left[T_{0}(x)-T_{\infty}\right] d x}{\int_{0}^{L} \rho C_{P} \theta_{n}(x) \theta_{n}(x) d x}$

If possible set the initial temperature profile as follows.

$T_{0}(x)=T_{c}^{0} f_{c}(x)+T_{s}^{0} f_{s}(x)$

Where $\mathrm{T}_{\mathrm{C}}{ }^{0}$ is the initial temperature at the center of the product and $\mathrm{T}_{\mathrm{s}}{ }^{0}$ is the initial temperature on the surface of the product. The functions $f_{c}(x)$ y $f_{s}(x)$ can be approximated as quadratic equations, as will be shown later.

Then the solution of the conservation of heat equation (14) is expressed as:

$T=A_{0}(x, t)+A_{c}(x, t) T_{c}^{0}+A_{s}(x, t) T_{s}^{0}$

Where:

$$
\begin{gathered}
A_{0}(x, t)=T_{\infty}-T_{\infty} \sum\left[\frac{\int_{0}^{L} \rho C_{P} \theta_{n}(x) d x}{\int_{0}^{L} \rho C_{P} \theta_{n}(x) \theta_{n}(x) d x}\right] e^{-\lambda_{n}^{2} t} \\
A_{c}(x, t)=\sum\left[\frac{\int_{0}^{L} \rho C_{P} \theta_{n}(x) f_{c}(x) d x}{\int_{0}^{L} \rho C_{P} \theta_{n}(x) \theta_{n}(x) d x}\right] e^{-\lambda_{n}^{2} t} \\
A_{s}(x, t)=\sum\left[\frac{\int_{0}^{L} \rho C_{P} \theta_{n}(x) f_{s}(x) d x}{\int_{0}^{L} \rho C_{P} \theta_{n}(x) \theta_{n}(x) d x}\right] e^{-\lambda_{n}^{2} t}
\end{gathered}
$$

To calculate the terms of $A_{n}$ we must have:

For the term in the denominator

$D_{n}=\int_{0}^{L} \rho C_{P} \theta_{n}(x) \theta_{n}(x) d x=$

$\rho_{1} C_{P 1} \int_{0}^{l} \theta_{n, 1}(x) \theta_{n, 1}(x) d x+\rho_{2} C_{P 2} \int_{l}^{L} \theta_{n, 2}(x) \theta_{n, 2}(x) d x$

In general, we would have that (the limits used in the integral as $L_{2}$ and $L_{1}$ to give generality and treat the two integrals that appear on the right side of equation 40):

$$
\begin{aligned}
& r_{i}^{2} \int_{L_{1}}^{L_{2}} \sin ^{2}\left(\frac{\lambda_{n}}{\sqrt{\alpha_{i}}} x+\phi_{n, i}\right) d x= \\
& \frac{r_{i}^{2}}{2}\left[\left(L_{2}-L_{1}\right)-\frac{\sqrt{\alpha_{i}}}{2 \lambda_{n}}\left[\sin \left(2 \frac{\lambda_{n}}{\sqrt{\alpha_{i}}} L_{2}+2 \phi_{n, i}\right)-\sin \left(2 \frac{\lambda_{n}}{\sqrt{\alpha_{i}}} L_{1}+2 \phi_{n, i}\right)\right]\right]
\end{aligned}
$$

As the functions $\mathrm{f}_{\mathrm{c}}(\mathrm{x})$ y $\mathrm{f}_{\mathrm{s}}(\mathrm{x})$ used in equation 37 are approximated as quadratic equations, it is necessary to express how the integrals of the numerator terms would be in equations 39. Then the result of these integrals for constant, linear and quadratic terms of a quadratic equation is shown.

For a constant term in the numerator we would have: 
$\int_{L_{1}}^{L_{2}} \theta_{n, i}(x) d x=$

$r_{i} \frac{\sqrt{\alpha_{i}}}{\lambda_{n}}\left[\cos \left(\frac{\lambda_{n}}{\sqrt{\alpha_{i}}} L_{1}+\phi_{n, i}\right)-\cos \left(\frac{\lambda_{n}}{\sqrt{\alpha_{i}}} L_{2}+\phi_{n, i}\right)\right]$

For a linear term in the numerator we would have:

$\int_{L_{1}}^{L_{2}} x \theta_{n, i}(x) d x=\left(r_{i} \frac{\alpha_{i}}{\lambda_{n}^{2}}\right)\left[\sin \left(\frac{\lambda_{n}}{\sqrt{\alpha_{i}}} L_{2}+\phi_{n, i}\right)-\sin \left(\frac{\lambda_{n}}{\sqrt{\alpha_{i}}} L_{1}+\phi_{n, i}\right)-\right.$

$\left.\frac{\lambda_{n}}{\sqrt{\alpha_{i}}} L_{2} \cos \left(\frac{\lambda_{n}}{\sqrt{\alpha_{i}}} L_{2}+\phi_{n, i}\right)+\frac{\lambda_{n}}{\sqrt{\alpha_{i}}} L_{1} \cos \left(\frac{\lambda_{n}}{\sqrt{\alpha_{i}}} L_{1}+\phi_{n, i}\right)\right]$

For a quadratic term in the numerator we would have:

$\int_{L_{1}}^{L_{2}} x^{2} \theta_{n, i}(x) d x=\left(r_{i} \frac{\sqrt{\alpha_{i}^{3}}}{\lambda_{n}^{3}}\right)\left[2 \cos \left(\frac{\lambda_{n}}{\sqrt{\alpha_{i}}} L_{2}+\phi_{n, i}\right)-\right.$

$2 \cos \left(\frac{\lambda_{n}}{\sqrt{\alpha_{i}}} L_{1}+\phi_{n, i}\right)+2\left(\frac{\lambda_{n}}{\sqrt{\alpha_{i}}} L_{2}\right) \sin \left(\frac{\lambda_{n}}{\sqrt{\alpha_{i}}} L_{2}+\phi_{n, i}\right)-$

$2\left(\frac{\lambda_{n}}{\sqrt{\alpha_{i}}} L_{1}\right) \sin \left(\frac{\lambda_{n}}{\sqrt{\alpha_{i}}} L_{1}+\phi_{n, i}\right)-\left(\frac{\lambda_{n}}{\sqrt{\alpha_{i}}} L_{2}\right)^{2}$

$\left.\cos \left(\frac{\lambda_{n}}{\sqrt{\alpha_{i}}} L_{2}+\phi_{n, i}\right)+\left(\frac{\lambda_{n}}{\sqrt{\alpha_{i}}} L_{1}\right)^{2} \cos \left(\frac{\lambda_{n}}{\sqrt{\alpha_{i}}} L_{1}+\phi_{n, i}\right)\right]$

What follows is to indicate the initial temperature profile that you should have at the beginning of the cooling stage. That is, define the equations $f_{s}(x)$ and $f_{c}(x)$.

Let's suppose our case where the product is heated first and then the cooling is carried out. Seeing the heating stage, since it is the one that will provide the initial condition for the cooling stage, which is where the surface temperatures are being measured. Before the product enters the heating stage, it is considered that it is entering at a uniform temperature, but when the product enters the cooling stage it will not be at a uniform temperature, because during heating the outer layers are the first to receive heat, which is subsequently transported into the product, transport that could not be carried out if there were no temperature gradient in the product. If the product came out of heating at a uniform temperature, it would not make sense to make these calculations, since the surface measurement would correspond to that of the center, but this is not the case, because the temperatures are different.

We can check if the profile can approach a quadratic profile after heating, we can enter the equations already given, (here we can do $B=0$, and we would have a uniform product temperature and see how at the end of heating the profile temperature approaches the quadratic). The Figure $\mathbf{2}$ shows the evolution of the temperature during the heating stage. For this case, the temperature profile was calculated for a typical case of baking cookies.

In Figure 2 it can be seen that the temperature profile inside, can be assumed without much error that it is a quadratic profile with a step at the interface of the product and the silicone plate, after a heating time of 5 minutes, a heating time longer than 30 min still shows a quadratic profile with the step, which decreases with longer heating times.

We are going to put how the evolution of heating would be, starting with a product at uniform temperature, in the Figure $\mathbf{2}$ it would be the internal

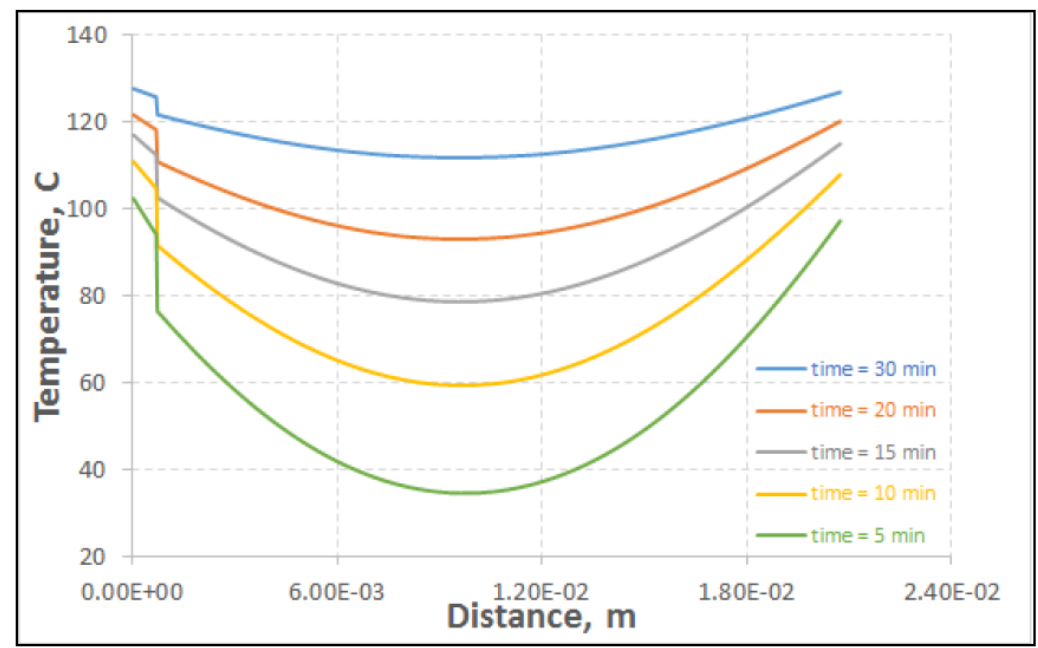

Figure 2: Evolution of temperature profile during the heating stage. 
temperature calculated from the resolution of the heat conservation equation. With more time this approach is getting closer, until it is getting closer and closer to quadratic profile; the experiments were carried out until obtaining a situation of this type.

The initial temperature profile in the region corresponding to the silicone plate is:

$$
\begin{gathered}
0 \leq x<l \\
f_{s}(x)=1+\frac{k_{2}}{k_{1}}\left[-1+4 \frac{l}{L}+\left(-3+4 \frac{l}{L}\right) a_{s}\right]\left(\frac{x}{L}\right) \\
f_{c}(x)=\frac{k_{2}}{k_{1}}\left[4\left(1-2 \frac{l}{L}\right)+\left(-3+4 \frac{l}{L}\right) a_{c}\right]\left(\frac{x}{L}\right)
\end{gathered}
$$

where:

$$
\begin{gathered}
a_{s}=\frac{1+\frac{l}{L}\left(1-\frac{k_{2}}{k_{1}}\right)-2\left(\frac{l}{L}\right)^{2}\left(1-2 \frac{k_{2}}{k_{1}}\right)}{1-3 \frac{l}{L}\left(1-\frac{k_{2}}{k_{1}}\right)+2\left(\frac{l}{L}\right)^{2}\left(1-2 \frac{k_{2}}{k_{1}}\right)} \\
a_{c}=\frac{-4 \frac{l}{L}\left(1-\frac{k_{2}}{k_{1}}\right)+4\left(\frac{l}{L}\right)^{2}\left(1-2 \frac{k_{2}}{k_{1}}\right)}{1-3 \frac{l}{L}\left(1-\frac{k_{2}}{k_{1}}\right)+2\left(\frac{l}{L}\right)^{2}\left(1-2 \frac{k_{2}}{k_{1}}\right)}
\end{gathered}
$$

While in the area corresponding to the product

$$
l<x \leq L
$$

we obtain:

$$
\begin{aligned}
& f_{s}(x)=a_{s}+\left[-1-3 a_{s}\right]\left(\frac{x}{L}\right)+\left[2+2 a_{s}\right]\left(\frac{x}{L}\right)^{2} \\
& f_{c}(x)=a_{c}+\left[4-3 a_{c}\right]\left(\frac{x}{L}\right)+\left[-4+2 a_{c}\right]\left(\frac{x}{L}\right)^{2}
\end{aligned}
$$

\section{INVERSE HEAT TRANSFER PROBLEM SOLVING APPROACH}

\subsection{Without Considering the Delay Time in the Measurement}

Let's go to the IHCP problem statement. We have our initial condition of the temperature profile at the start of cooling. From this we can know $\mathrm{T}_{\mathrm{s}}{ }^{0}$ because we are measuring it, but we do not know $T_{c}{ }^{0}$, which is the temperature at the center of the product, which is what we want to know based on the surface temperature readings.

Recalling the equation 38 (We don't put all the terms in the series, just a few, because the higher terms are multiplied by exponentials that decrease faster as time passes) and evaluating is in the position where $\mathrm{x}=\mathrm{L}$, we have:

$T(L, t)=A_{0}(L, t)+A_{s}(L, t) T_{s}^{0}+A_{c}(L, t) T_{c}^{0}$

Then we must treat the least squares problem, comparing it with the measurements obtained by thermography $T_{s, \text { med }}$

$S=\sum\left(T_{s, \text { med }}-T(L, t)\right)^{2}$

Where making the following changes to the equations

$$
\begin{gathered}
y=T_{s, \text { med }}-A_{0}(L, t)-A_{s}(L, t) T_{s}^{0} \\
x=A_{c}(L, t) \\
m=T_{c}^{0}
\end{gathered}
$$

It comes to the following

$S=\sum(y-m x)^{2}$

Minimizing the summation of squared errors expression

$m=\frac{\sum y_{i} x_{i}}{\sum x_{i} x_{i}}$

\subsection{Considering the Delay Time in the Start of the Measurement}

For the case where there was a delay in the measurement, the algorithm would have change to take this effect into account. Starting with a delay at the beginning of the measurement should be avoided, but in the event that it occurs, it would be necessary to quantify how the method would change and how long the delay would be tolerable. A delay in the measurement time alters the assumption of having a quadratic profile, since in that course of time the surface layers would cool down, giving a different profile to the quadratic.

Then we would have:

$$
\begin{aligned}
& T_{c}=T\left(\frac{L}{2}, t_{r e t}\right)=A_{0}\left(\frac{L}{2}, t_{r e t}\right)+A_{c}\left(\frac{L}{2}, t_{r e t}\right) T_{c}^{0}+A_{s}\left(\frac{L}{2}, t_{r e t}\right) T_{s}^{0} \\
& T_{s}=T\left(L, t_{r e t}\right)=A_{0}\left(L, t_{r e t}\right)+A_{c}\left(L, t_{r e t}\right) T_{c}^{0}+A_{s}\left(L, t_{r e t}\right) T_{s}^{0}
\end{aligned}
$$


Solving we would obtain:

$T_{c}^{0}=a_{c o}+a_{c c} T_{c}+a_{c s} T_{s}$

$T_{s}^{0}=a_{s o}+a_{s c} T_{c}+a_{s s} T_{s}$

Then the equation 38 can be written as:

$$
\begin{aligned}
& T(x, t)=A_{0}(x, t)+A_{c}(x, t)\left[a_{c o}+a_{c c} T_{c}+a_{c s} T_{s}\right] \\
& +A_{s}(x, t)\left[a_{s o}+a_{s c} T_{c}+a_{s s} T_{s}\right]
\end{aligned}
$$

Then

$$
\begin{aligned}
& T(x, t)=\left[A_{0}(x, t)+A_{c}(x, t) a_{c o}+A_{s}(x, t) a_{s o}\right]+ \\
& {\left[A_{c}(x, t) a_{c c}+A_{s}(x, t) a_{s c}\right] T_{c}+\left[A_{c}(x, t) a_{c s}+A_{s}(x, t) a_{s s}\right] T_{s}}
\end{aligned}
$$

The equations 50 obtained previously, are now expressed as:

$y^{+}=T(L, t)-\left[A_{0}(x, t)+A_{c}(x, t) a_{c o}+A_{s}(x, t) a_{s o}\right]$

$-\left[A_{c}(x, t) a_{c s}+A_{s}(x, t) a_{s s}\right] T_{s}$

$x^{+}=\left[A_{c}(x, t) a_{c c}+A_{s}(x, t) a_{s c}\right]$

\section{Equation 56 as}

$y^{+}=m x^{+}$
The parameter $\mathrm{m}$ can be obtained using least squares techniques, as

$m=\frac{\sum y^{+} x^{+}}{\sum x^{+} x^{+}}$

We have the following equation.

$m=T_{c}$

In this case $m$ is the predicted value for $T_{c}$ that corresponds to the start of the measurements, if the value of $T_{c}{ }^{0}$ is required, which is the corresponding value at a time before the beginning of the measurement, it can be calculated based on equation 54.

\section{EXPERIMENTAL DEVELOPMENT}

The following equipment was used to carry out the experiments: a thermographic camera Raytek Thermo $V_{\text {View }}{ }^{\mathrm{TM}} \mathrm{Pi} 20$ for surface temperature detection working in the spectral range between 8 up to $14 \mu \mathrm{m}$, the internal temperature was obtained with a type $J$ thermocouple connected to the data acquisition card National Instruments SCXI-1112 for 8 channels, which is assembled with a NI PXI-8106 Embedded Controller from National Instruments. The heating of the pieces were obtained with a convection oven.

Figure 3 shows the arrangement of the equipment for the acquisition of temperatures during cooking of

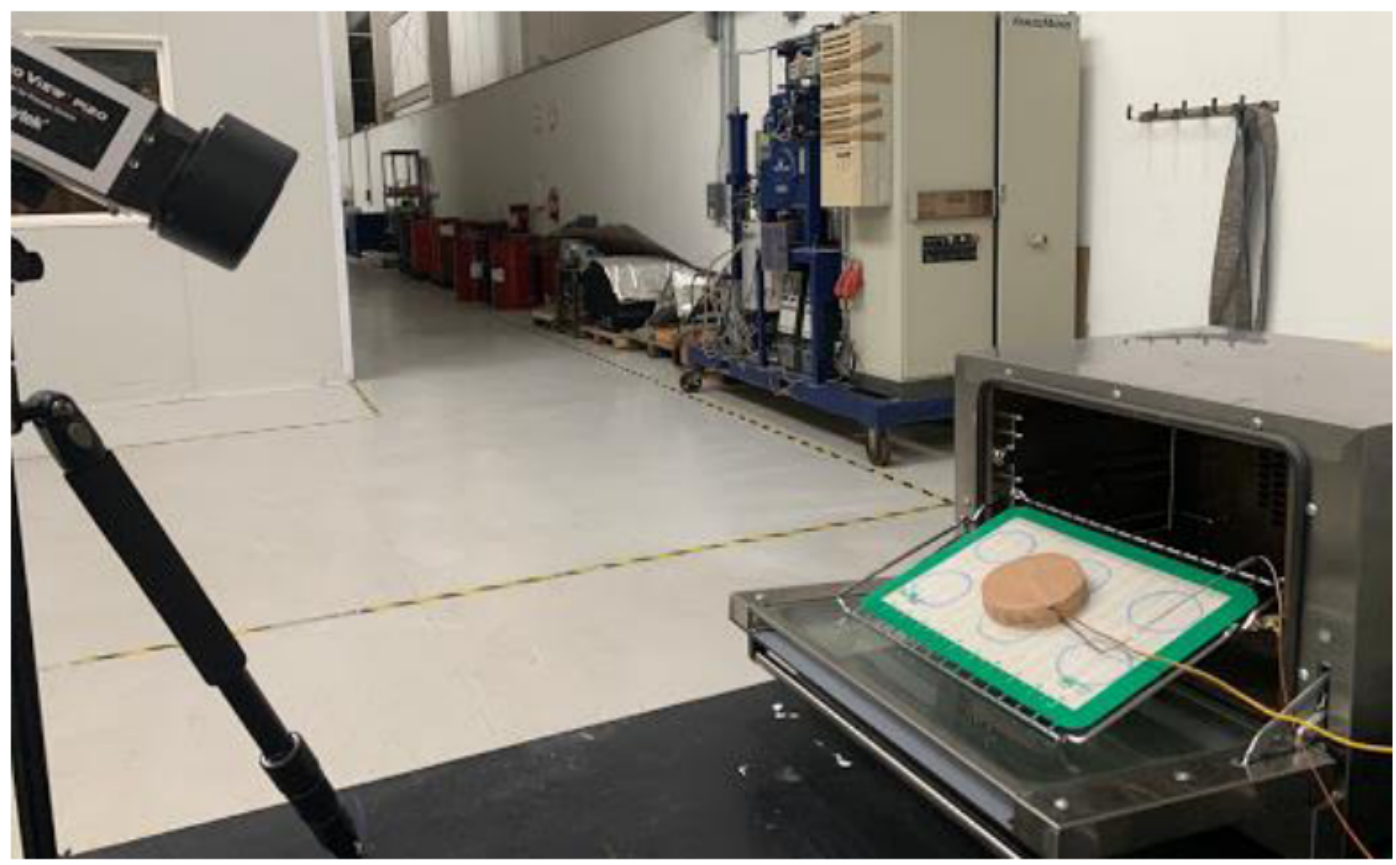

Figure 3: Experimental setup. 
products. In each of the experiments, the product was heated to a certain temperature, a fixed cooking time was maintained (30 min), after which it was removed from the oven and the central temperature was monitored with the thermocouple and the surface temperature with the thermal camera. Archived data were subsequently analyzed with a program coded in MATLAB. Geometric measurements of the products (length and diameter) were taken.

\section{RESULTS}

Thermophysical properties of cookies and baking silicon plate are shown on Table 1 and were obtained by correlations as function of their composition [9, 10].

Table 1: Thermophysical Properties of Product and Silicon, and Thickness of Silicon

\begin{tabular}{|c|c|}
\hline Property & Value \\
\hline \multicolumn{2}{|c|}{ Product } \\
\hline density, $\mathrm{kg} / \mathrm{m} 3$ & 1222.12 \\
\hline Specific Heat, J/kg K & 2940 \\
\hline Thermal conductivity, $\mathrm{W} / \mathrm{mK}$ & 0.3011 \\
\hline \multicolumn{2}{|c|}{ Silicon } \\
\hline density, $\mathrm{kg} / \mathrm{m} 3$ & 1300 \\
\hline Specific Heat, J/kg K & 1400 \\
\hline Thermal conductivity, $\mathrm{W} / \mathrm{mK}$ & 0.22 \\
\hline \multicolumn{2}{|c|}{ Parameters } \\
\hline Thickness, mm & 0.73 \\
\hline Thermal resistance, $\mathrm{m} 2 \mathrm{~K} / \mathrm{W}$ & 0.0005 \\
\hline
\end{tabular}

Operational parameters for both sets of experiments are presented on Table $\mathbf{2}$.

From the previous discussion, the effect of delay time in the determination of internal temperature had to be included, as well as considering the thermocouple measurement point inside the product to determine the heat transfer coefficient.

The heat transfer coefficient values and thermal resistance from Table 2 were obtained by minimizing the difference between measured temperature values and model temperatures.

The Figure 4 shows the measured temperature and the calculated temperature corresponding to experiments for cooling of cookies.

The Figures 5 and 6 show a better comparison between the measured temperatures and the calculated ones for experiments. A linear relationship can be shown in the figures.

The Figures 7 shows the graphs of " $y$ " vs " $x$ " like as defined in the equations 57 and 58 . The mathematical model predicts that a line straight can be obtained. This prediction is found by examining the graphs. The slope of the line is equal to the core temperature of the product at time equals zero as shown in the equation 61.

The Table 3 shows a comparison between the estimated temperature by the algorithm and the measured temperature. The parameter " $\mathrm{m}$ " gives the core temperature, using the resolution of the heat transfer equation; the temperature at the measurement

Table 2: Operational Parameters used in the Experiments

\begin{tabular}{|c|c|c|c|}
\hline Parameter & Test 1 & Test 2 & Test 3 \\
\hline Heating time, min & 20 & 20 & 20 \\
\hline Heat transfer coeficient, $\mathrm{W} / \mathrm{m} 2 \mathrm{~K}$ & 30 & 20.5 & 31.8 \\
\hline Enviroment temperature, $\mathrm{C}$ & 22 & 23 & 25 \\
\hline Retard time, seg & 21.3 & 22 & 18.5 \\
\hline \multicolumn{4}{|l|}{ Dimensiones } \\
\hline Thickness, mm & 18.37 & 17.87 & 19.38 \\
\hline Diameter, mm & 64.77 & 63.78 & 64.7 \\
\hline $\begin{array}{l}\text { point of measurement of temperature taken from superior } \\
\text { part, } \mathrm{mm}\end{array}$ & 12.46 & 8.58 & 11.54 \\
\hline
\end{tabular}



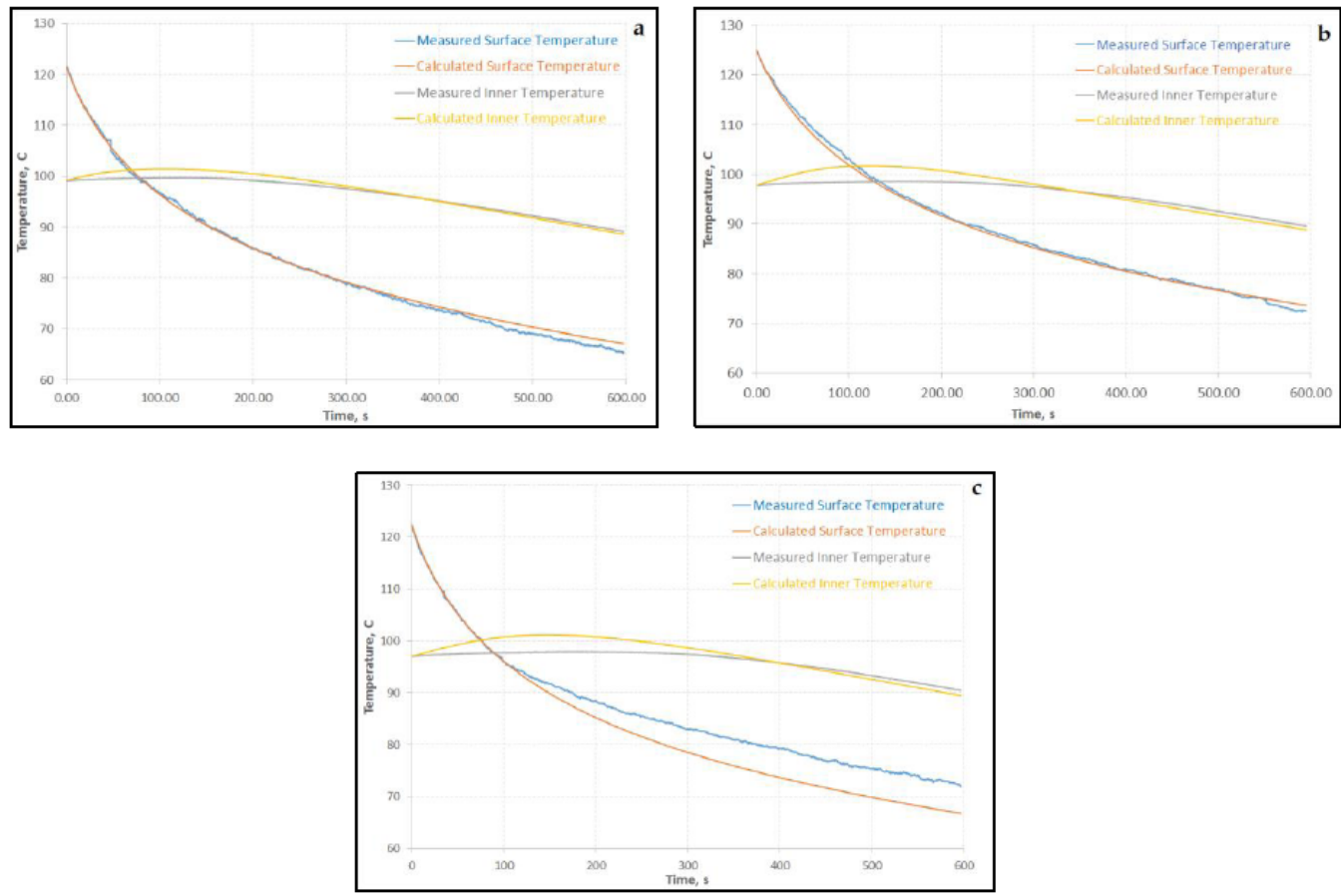

Figure 4: measured temperatures and calculated temperatures.

a) Test 1, b) Test 2 ad c) Test 3 .
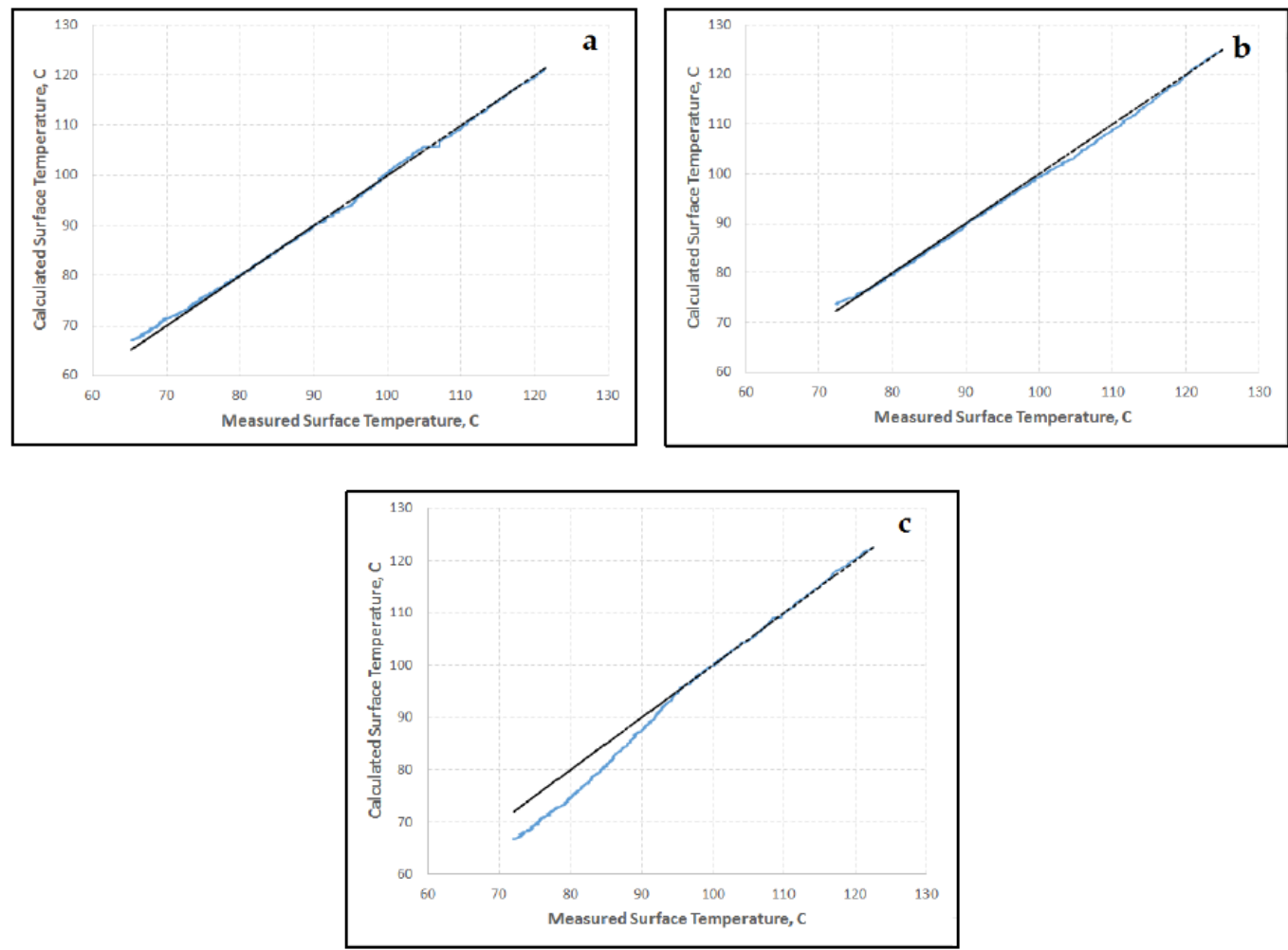

Figure 5: Comparison between measured and calculated surface temperatures.

a) Test 1, b) Test 2 and c) Test 3 .

point of the inserted thermocouple is calculated for comparison purposes.
Maintaining high production speed would require that the time to obtain the surface temperature curve 

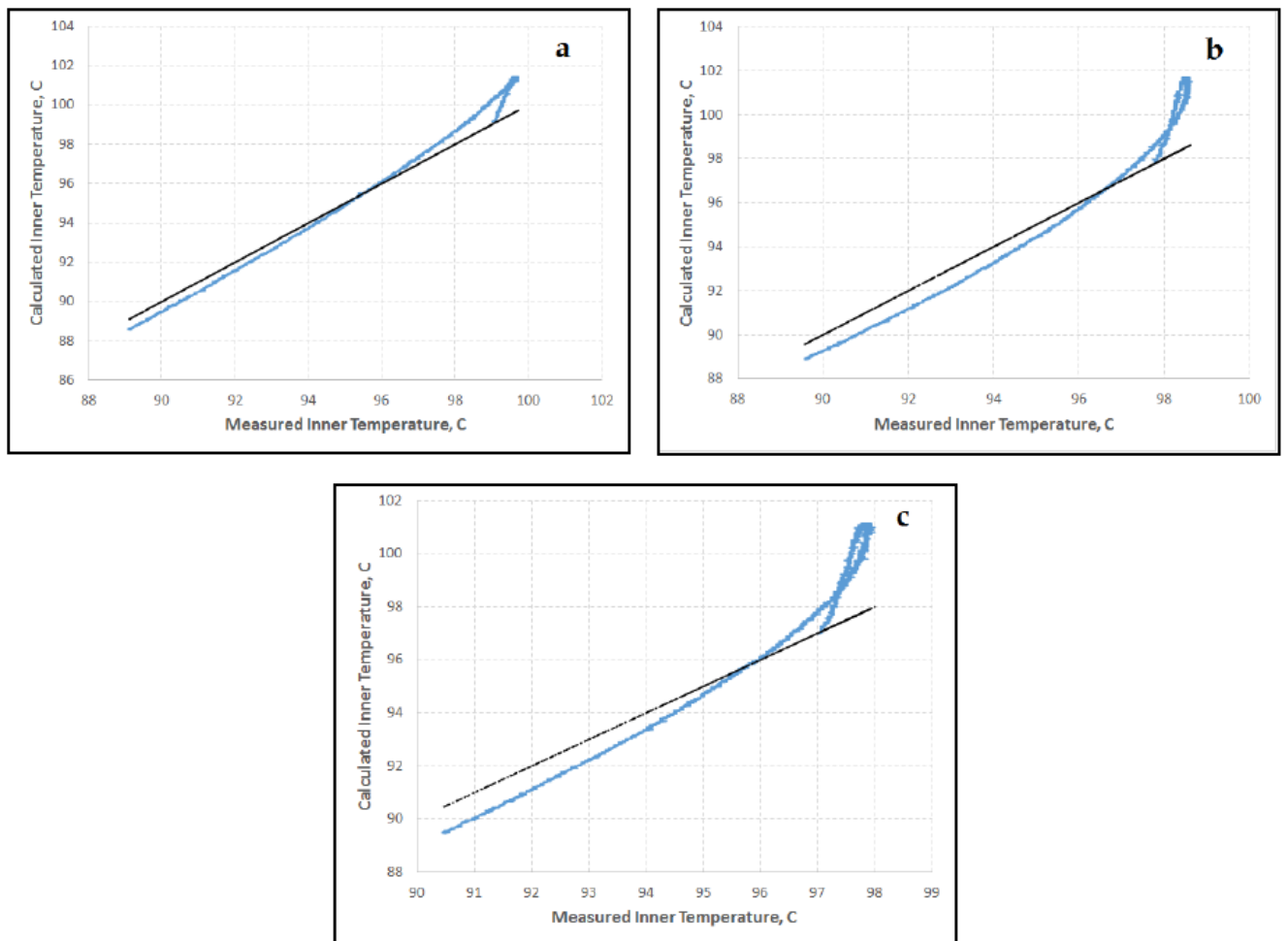

Figure 6: Comparison between measured and calculated inner temperatures.

a) Test 1, b) Test 2 and c) Test 3 .
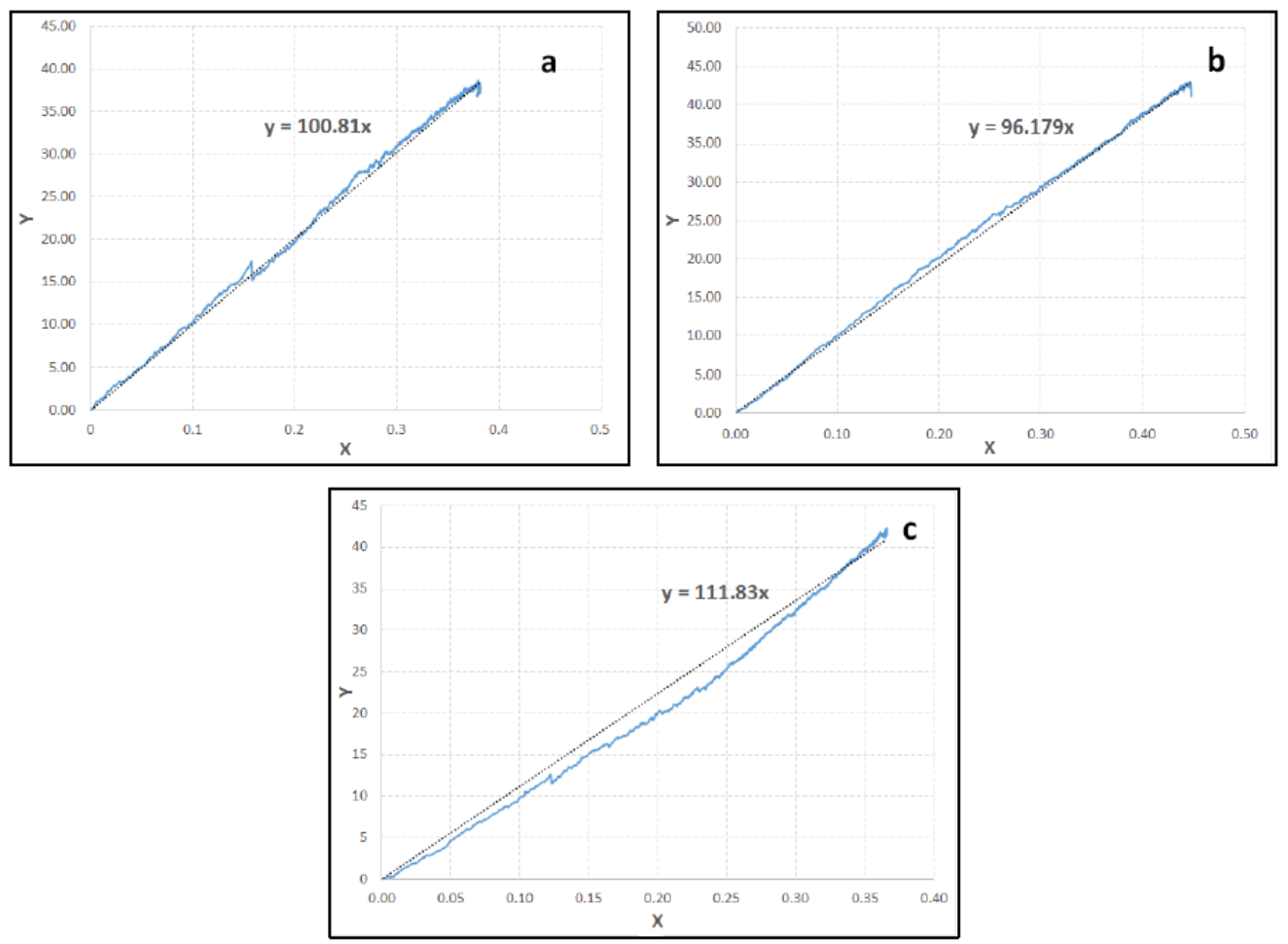

Figure 7: Graphs of $y$ vs $x$ defined in the equations 54 for experiments of cooling.

a) Test 1, b) Test 2 and c) Test 3 .

should not be too long. Table 4 shows the result for the collection times. internal temperature estimation for different data 
Table 3: Comparison between the Estimated Temperature by the Algorithm and the Measured Temperature

\begin{tabular}{|c|c|c|c|c|}
\hline Number of Test & Slope $\mathbf{~ m},{ }^{\circ} \mathbf{C}$ & $\begin{array}{c}\text { Point of } \\
\text { Measurement, } \mathbf{~ m m}\end{array}$ & $\begin{array}{c}\text { Temp. Measured }{ }^{\circ} \mathbf{C}, \\
\mathbf{t}=\mathbf{0 ~ s}\end{array}$ & $\begin{array}{c}\text { Temperature Predicted by Model at Point of } \\
\text { Measurement }\end{array}$ \\
\hline 1 & 100.81 & 12.46 & 99.136 & 96.81 \\
\hline 2 & 96.179 & 8.58 & 97.823 & 98.36 \\
\hline 3 & 111.83 & 11.54 & 96.987 & 110.33 \\
\hline
\end{tabular}

Table 4: Comparison between the Estimated Temperature by the Algorithm at Different Times and the Measured Temperature

\begin{tabular}{|c|c|c|c|}
\hline & \multicolumn{3}{|c|}{ Number of Test } \\
\hline $\begin{array}{c}\text { Temperature predicted } \\
\text { using data from curve at }\end{array}$ & $\mathbf{1}$ & $\mathbf{2}$ & $\mathbf{3}$ \\
\hline \hline $\mathrm{t}=20 \mathrm{~s}$ & 100.47 & 101.19 & 90.27 \\
\hline $\mathrm{t}=30 \mathrm{~s}$ & 101.08 & 102.09 & 93.18 \\
\hline $\mathrm{t}=60 \mathrm{~s}$ & 98.63 & 103.46 & 95.89 \\
\hline
\end{tabular}

\section{DISCUSSION}

Advantages of this technique are: it is not invasive, nor destructive, it can determine the temperature of a set of objects; in contrast with traditional methods which include the insertion of a thermocouple on a limited number of samples that must be discarded; since this method was obtained by solution of fundamental equations of heat transfer, it is possible to be use with objects with different properties, dimensions or cooling conditions. Even though the method is complex, irregular geometries have not yet been treated and it requires constant thermal properties and coefficient, the agreement between the model and experimental results show that solving of the equations and the considerations made were well established as explained and developed on the body of the article. To our knowledge there is only one more group of researchers working on the estimation of inner temperature by infrared thermography [2], the main difference between these works is that they work with chicken irregular geometry samples and solve the problem by using neural networks techniques, due to the use of irregular geometries they could not solve the problem by fundamental heat transfer equations which increase the number of test that must be perform to get good agreement with their model and its application is limited to that particular case.

When the mathematical model was developed, a perfect thermal contact was assumed but as it progressed it was noticed that it had to be corrected considering thermal interfacial resistance between the test material and the cooking surface used. Another of the critical variables that had to be considered was the delay time between the samples cooling begging and temperature measurements, it is important to consider this as it also has an effect on the established model, modifying the initial profile of the internal temperature, moving it away from the quadratic profile that characterizes it. As mentioned, at the beginning the cooling profile is closely quadratic, but over time, the exposure of the surface makes its temperature colder and therefore affects the internal temperature profile. To be considered for an industrial application, it should be sought that the delay time be the minimum possible, as it is known by the IHCP literature that the error in the estimation is very sensitive to the measurement conditions, causing it to increase exponentially [7].

Considering the above, some parameters of the model were adjusted to achieve agreement in the cooling curves (Figures 4 and 5) this allowed us to determine the internal temperature with a tolerable error $\left(\sim 2^{\circ} \mathrm{C}\right)$. In Figure 7 it can be seen that the cooling curve can be expressed in terms of the variables $X$ and $Y$ defined in equations 57 and 58, where it is obtained that the plot can be approximated to a straight line, allowing the estimation of the internal temperature according to the established mode, the error in the estimation of the internal temperature decreases as it gets closer to the straight line, in the first two tests there is an error of $1.7^{\circ} \mathrm{C}$. If it is wanted to be taken for an industrial application, the reading time of the surface temperature cooling curve must be optimized in order to avoid a production delay, avoiding long reading times (data collection), but not too short that affect the reliability of the model and the error in the estimation increases; Table 4 shows the values obtained at different data collection times, showing that the model is sensitive to this parameter; which leads to the development of an uncertainty model in order to optimize the data collection time. 


\section{CONCLUSION}

An IHCP methodology is developed to determine the internal temperature in bodies from Surface temperature measurements by infrared thermometry. The method is based on fundamental mechanism of heat transfer. Good agreement is obtained comparing between model results with those obtained from the experiments.

The general limitation of the method to determinate the core temperature from superficial temperatures is the time required for the heat to be transmitted from the central area to the outside. This time depends on the thermal properties, dimensions of the product and the cooling conditions. There is a time window where the temperature measurement must be performed to obtain adequate results. A long measurement time would increase the process time and therefore decrease the production rate. While a short measurement time would not allow the effect of the inner layers to be felt in the surface temperature measurement, which would result in a greater error in the determination of internal temperature. Among the variables that can affect the range of the window of measurement time are product thickness, thermophysical properties of the material and cooling conditions. It would be easier to appreciate the effect of these variables as a function of dimensionless numbers. An important number to define the range of measurement time is the Fourier number, which shows that the minimum measurement time would increase in products of greater thickness and a material with less thermal diffusivity. These parameters that would make the time in which the effect of the internal temperature felt on the surface temperature reading will move to higher values.

Another important aspect is the lag time between the start of temperature reading and the beginning of cooling step. At the end of the product's heating stage, there is a quadratic profile in the temperature where the external part is the hottest, and the coldest is inside the product, when the product goes to the cooling stage, this temperature profile is changing, the surface layer of the product is cooling, in this area the temperature is falling, therefore the temperature profile is moving away from the initial quadratic profile, as this difference progresses, which means that if there is a delay between the time at which the temperature is being tracked and the start of the cooling stage would give an error in the procedure. Taking into account the delay time for starting the temperature measurement is important if it is necessary to improve the determination of internal temperature, but it must be taken into account that this delay should not be excessive, because the error of determination of internal temperature will be increase.

\section{FUTURE WORK}

As already mentioned, the IHCP literature suggests that the error in the determination of the inner temperature is very sensitive to the measurement conditions, causing it to increase exponentially [7]. Among the variables that can affect the range of the window of measurement time are product thickness, thermophysical properties of the material and cooling conditions. It would be easier to appreciate the effect of these variables as a function of dimensionless numbers. An important number to define the range of measurement time is the Fourier number, which shows that the minimum measurement time would increase in products of greater thickness and a material with less thermal diffusivity. These parameters that would make the time in which the effect of the internal temperature felt on the surface temperature reading will move to higher values. To verify the above, experimentation and a model that expresses the error due to the uncertainty of the parameters is required.

\section{REFERENCES}

[1] Bin Yin, Ling Ling Cao, inventor; Koninklike Philips NV. assignee. Apparatus and Method for Determining Core Temperature of Food. United States Patent US20150362378. 2015 December.

[2] Juan G. Ibarra, Yang Tao, Hongwei Xin. Combined IR Imaging-Neural Network Method for the Estimation of Internal Temperature in Cooked Chicken Meat. Opt. Eng 2000; 39(11): 3032-3038.

https://doi.org/10.1117/1.1314595

[3] Ling Ma, An Infrared and Laser Range Imaging System for Non-Invasive Estimation of Internal Cooking Temperature in Poultry Fillets. Faculty of the Graduate School of the University of Maryland, College Park 2003.

[4] A. Bendada and KT. Nguyen. Estimation of the initia Temperature Profile and the Heat Transfer Coefficient in Polymer Processing by an Inverse Method. Proceedings of the 3rd International Conference on Inverse Problems in Engineering; 1999: Port Ludlow, Washington, USA; 1999: $p$. 1-7.

[5] Marcus Shelby, Kevin Horton, Harry Hall, inventor; Grupo Petrotemex SA de CV, assignee. Method and Device for Predicting Temperature Profiles Throughout the Thickness of a Polymer Preform. United States Patent US20040024560A1. February 2004.

[6] Dean Anthony, Daipayan Sarkar, Ankur Jain. Non-invasive, Transient Determination of the Core Temperature of a Heat Generating Solid Body. Sci. Rep 2016; 6: 35886. https://doi.org/10.1038/srep35886

[7] M. Necati Ozisik, H.R. Orlande. Inverse Heat Transfer, Fundamentals and applications. Taylor \& Francis 2000.

[8] Eduardo Macias Avila and Yaneth Aguilar Diaz. Inner Food Temperature Estimation by IHCP Methodology on Surface 
Temperature Measurements obtained by Thermography. International Journal of Food Processing Technology 2020;

6: $1-9$

https://doi.org/10.15379/2408-9826.2020.06.01.01

[9] American Society of Heating, Refrigeration and Air Conditioning Engineers. AHSRAE Handbook Refrigeration. In: American Society of Heating, Refrigeration and Air
Conditioning Engineers. Thermal Properties of Food 2006; p. 9.1-9.31.

[10] Esther Bejarano, Martha Bravo, Mayola Huaman, Clotilde Huapaya, Amalia Roca, Edith Rojas. Tabla de composición de alimentos industrializados. Ministerio de salud, Centro nacional de Alimentación y Nutrición. Lima, Perú 2002.

http://dx.doi.org/10.15379/2408-9826.2021.07.01.01

(c) 2021 Ávila and Díaz; Licensee Cosmos Scholars Publishing House.

This is an open access article licensed under the terms of the Creative Commons Attribution Non-Commercial License (http://creativecommons.org/licenses/by-nc/3.0/), which permits unrestricted, non-commercial use, distribution and reproduction in any medium, provided the work is properly cited. 